\title{
A Tangent Linear Approximation of the Ignition Delay Time. I: Sensitivity to Rate Parameters
}

\author{
Saja Almohammadi ${ }^{\mathrm{a}}$, Mireille Hantouche ${ }^{\mathrm{b}}$, Olivier P. Le Maîtrec ${ }^{\mathrm{c}}$, Omar M. Knio ${ }^{\mathrm{a}, *}$ \\ ${ }^{a}$ Division of Computer, Electrical, and Mathematical Sciences and Engineering, King Abdullah University of Science and \\ Technology, Thuwal, Saudi Arabia \\ ${ }^{b}$ Division of Physical Science and Engineering, King Abdullah University of Science and Technology, Thuwal, Saudi Arabia \\ ${ }^{c}$ Centre de Mathématiques Appliquées, CNRS, Inria, École Polytechnique, 91128 Palaiseau, France
}

\begin{abstract}
A tangent linear approximation is developed to estimate the sensitivity of the ignition delay time with respect to individual rate parameters in a detailed chemical mechanism. Attention is focused on a gas mixture reacting under adiabatic, constant-volume conditions. The uncertainty in the rates of elementary reactions is described in terms of uncertainty factors, and are parametrized using independent canonical random variables. The approach is based on integrating the linearized system of equations governing the evolution of the partial derivatives of the state vector with respect to individual random variables, and a linearized approximation is developed to relate the ignition delay sensitivity to the scaled partial derivatives of temperature. The efficiency of the approach is demonstrated through applications to chemical mechanisms of different sizes. In particular, the computations indicate that for detailed reaction mechanisms the TLA leads to robust local sensitivity predictions at a computational cost that is order-of-magnitude smaller than that incurred by finite-difference approaches based on one-at-a-time rate perturbations.
\end{abstract}

Keywords: local sensitivity; rate coefficients; tangent linear approximation; uncertainty factor; Jacobian

\section{Introduction}

The ignition delay time of a mixture reacting adiabatically at constant volume is an important quantity of interest (QoI) used to characterize the reactivity of fuels and fuel blends, and to assess the suitability of comprehensive reaction models (e.g. [1-7]). A key aspect of such analyses concerns the dependencies of the ignition delay time on uncertainties in rate parameters. These dependencies have received much interest in the literature, and have motivated the development of uncertainty quantification (UQ) techniques and their application to conduct global sensitivity analyses, and parameter calibration, reduction strategies, and experimental design (e.g. [7-17]).

Local sensitivity analyses have been effectively used to analyze the dependence of the ignition delay time on rate parameters [18-22], traditionally as a means to rank important reactions. The availability of local sensitivity estimates for a sufficiently large sample of the uncertain reaction rates can also be exploited to identify active subspaces [23-26], and subsequently construct suitable reduced dimensionality surrogates. As active subspace methods require the estimation of local sensitivities for each realization of uncertain reaction rates, application of conventional finite-difference methodologies (e.g. [27-33]) would be prohibitively expensive. This is the case because in the finite-difference approach the reaction rates are individually perturbed, and the response of the system is computed by applying the solver (e.g. [34-36]) for each perturbation. For a random input parameter vector of dimension $d$, this approach requires $d$ independent applications of the solver. When uncertainty in all elementary reactions are considered, the dimensionality $d$ typically coincides with the number of reactions, $N_{r}$. Consequently, one must resort to

*Corresponding author. Email: omar.knio@kaust.edu.sa 
alternative means of estimating local sensitivities, particularly defeating the $\mathrm{O}(\mathrm{d})$ cost of the brute force approach.

Several approaches have been suggested in the literature that offer to achieve such objective. In particular, tangent linear approximation (TLA) methodologies have been proposed (e.g. [18, 37]) that rely on integrating the evolution equations of the perturbed system. These methods yield the linearized time response of the state vector to individual perturbations of the parameters. The TLA approach has been combined in [38] with a logarithmic differentiation scheme, namely to relate the perturbed ignition delay time to the state vector perturbation, and consequently estimate the local sensitivity of the ignition delay time to individual perturbations of rate parameters. Recently, alternatives to the direct differentiation approach have also been proposed. Specifically, an adjoint based methodology was proposed in [39] to compute the evolution of the perturbed system, and was reported to yield dramatic improvement over the finite-difference approach. An optimization-based approach was proposed in [40] to estimate the local sensitivity of the ignition delay time to perturbations in reaction parameters. The approach is based on recasting the system as a boundary value problem (BVP) whose solution yields the ignition delay time perturbations. Experiences reported in [40] indicate that the BVP approach can result in order-of-magnitude reduction in computational time compared to the brute force approach.

In this work, we explore the application of a TLA approach to estimate the local sensitivity of the ignition delay time with respect to reaction parameters, at a dramatically smaller cost than that associated with the finite-difference technique as $d$ becomes large. Here, we restrict our attention to reaction rate parameters, and leave to follow on work the task of addressing sensitivities to variations in thermodynamic properties. Following a methodology to that proposed in $[18,37]$, we derive a dynamical system of equations that govern the evolution of infinitesimal perturbations of the state vector. We rely on a framework in which the uncertain rates are described by uncertainty factors, and parametrized using independent uniformlydistributed canonical random variables. We focus on the evolution of perturbations in the vector of canonical random variables, starting from a vanishing initial condition. In the resulting linearized system, the evolution of the perturbations takes the form of a path integral involving the Jacobian of the unperturbed solution, and the local derivatives of the unperturbed source term. Because the ignition delay is an implicit function of the solution, one needs to relate local sensitivities of the ignition delay time to the partial derivatives of the state vector, which can be obtained by integrating the linearized system. With the ignition delay time defined as the time needed for the temperature of the mixture to increase by a user-defined amount, a linearized approximation is introduced, providing an explicit relationship between the desired ignition delay time sensitivities and scaled temperature perturbations at the unperturbed ignition delay time. Briefly, our objectives are to (i) formulate a stochastic tangent linear approximation of local sensitivities of the solution of a gas mixture reacting adiabatically at constant volume, and (ii) explore whether the state sensitivities obtained by integrating the linearized system can be suitably exploited to estimate local sensitivities of the ignition delay time.

The paper is organized as follows. Key features of the proposed approach are introduced in section 2 . We start by summarizing the governing equations for the non-linear system (section 2.1) and then highlight the setup used to describe and parametrize the uncertain rates (section 2.2). In section 2.3, we state the equations of motion of the linearized system that govern the evolution of the partial derivatives of the state vector with respect to individual components of the uncertainty germ. A linearized approximation is then introduced in section 2.4 to relate local sensitivities of the ignition delay time to partial derivatives of temperature. In section 2.5 an analytical identity is derived that is later exploited to test or refine computed results. In section 3, we describe our approach used to integrate the TLA. Results of numerical experiments and performance assessments are reported in section 4. Major conclusions are highlighted in section 5.

\section{Formulation}

\subsection{Governing Equations}

In this section, we start by summarizing the governing equation for homogeneous combustion of a reacting gas mixture under adiabatic, constant-volume conditions $[36,41]$. We let $Y_{i}, i=1, \ldots, N_{s}$ denote the species 
mass fractions, $N_{s}$ denote the number of reacting species, $T$ denote the temperature, whereas $t$ is time. For a detailed chemical mechanism involving $N_{r}$ elementary reactions, the evolution of the species mass fractions and temperature is governed by the coupled system:

$$
\begin{aligned}
\frac{d Y_{i}}{d t} & =\frac{1}{\rho} \sum_{j=1}^{N_{r}} \dot{\omega}_{i}^{j}, \quad i=1, \ldots, N_{s} \\
\frac{d T}{d t} & =-\frac{\gamma}{\rho c_{p}} \sum_{i=1}^{N_{s}} h_{i}\left(\sum_{j=1}^{N_{r}} \dot{\omega}_{i}^{j}\right)+(\gamma-1) \frac{T W}{\rho} \sum_{i=1}^{N_{s}} \frac{1}{W_{i}}\left(\sum_{j=1}^{N_{r}} \dot{\omega}_{i}^{j}\right)
\end{aligned}
$$

where $\rho$ denotes the mixture density, $c_{p}$ is the heat capacity at constant pressure, $c_{v}$ is the heat capacity at constant volume, $\gamma \equiv c_{p} / c_{v}$ is the specific heat ratio, $\dot{\omega}_{i}^{j}$ is rate of production of species $i$ due to reaction $j$, $h_{i}$ is the enthalpy of species $i, W_{i}$ is the molecular weight of species $i$, whereas

$$
W \equiv\left(\sum_{i=1}^{N_{s}} \frac{Y_{i}}{W_{i}}\right)^{-1}
$$

A perfect gas mixture assumption is used [36], whereas standard-state thermodynamic properties are computed using NASA polynomials [42].

\subsection{Setup}

Our main objective is to estimate the sensitivity of the ignition delay time, $\tau$, with respect to infinitesimal perturbations in the stochastic germ parametrizing the uncertainty in individual reaction rates. Specifically, the uncertainty in the elementary reaction rates is assumed to be specified using uncertainty factors, $\mathrm{UF}_{j}$, $j=1, \ldots, N_{r}$. The corresponding reaction rate uncertainties are parametrized according to [43, 44] :

$$
A_{j}\left(\xi_{j}\right)=\mathrm{UF}_{j}^{\xi_{j}} \bar{A}_{j}
$$

where $A_{j}$ is the pre-exponent of reaction $j$, the $\xi_{j}$ are canonical random variables that are independent and uniformly distributed over $[-1,1]$, and $\bar{A}_{j}$ is the "nominal" pre-exponential of reaction $j$, i.e. $\bar{A}_{j}=$ $A_{j}\left(\xi_{j}=0\right)$. Further, we ignore uncertainties in thermodynamic parameters, and assume that variations of the forward and reverse rates of the $j$-th reaction are perfectly correlated.

Note that from (3) it immediately follows that

$$
\frac{\partial A_{j}}{\partial \xi_{j}}=\ln \left(\mathrm{UF}_{j}\right) A_{j}
$$

and in light of the assumptions stated above this leads to

$$
\frac{\partial \dot{\omega}_{i}^{j}}{\partial \xi_{j}}=\ln \left(\mathrm{UF}_{j}\right) \dot{\omega}_{i}^{j}(\boldsymbol{\xi}),
$$

where $\dot{\omega}_{i}^{j}(\boldsymbol{\xi})$ is the chemical production term obtained with the local pre-exponentials $A_{j}\left(\xi_{j}\right)$ from Eq. (3).

In the present setup, our goal is to develop an efficient methodology that enables us to estimate the local sensitivity of the ignition delay time, $\tau$, with respect to infinitesimal perturbations of individual components of the germ, $\boldsymbol{\xi}$, i.e., to determine the partial derivatives $\partial \tau / \partial \xi_{j}$, and/or the local sensitivity indices:

$$
S_{j}^{\tau} \equiv A_{j} \frac{1}{\frac{\partial A_{j}}{\partial \xi_{j}}} \frac{1}{\tau} \frac{\partial \tau}{\partial \xi_{j}} .
$$

Substituting (4) into (6), we obtain

$$
S_{j}^{\tau}=\eta_{j} \frac{1}{\tau} \frac{\partial \tau}{\partial \xi_{j}},
$$


where

$$
\eta_{j} \equiv \frac{1}{\ln \left(\mathrm{UF}_{j}\right)}, \quad j=1, \ldots, N_{r} .
$$

Remark 1. Note that, for the same infinitesimal change in the germ, a larger uncertainty factor will result in a larger perturbation of the pre-exponential parameter. This can be readily appreciated from (4). Consequently, the partial derivatives $\partial \tau / \partial \xi_{j}$ will also depend on the uncertainty factors $\mathrm{UF}_{j}$. In the definition of the local sensitivity indices (6), the effects of varying uncertainty factors are appropriately normalized. This is reflected in the fact that (7) holds regardless of the selected values of the uncertainty factors.

Remark 2. The expression of the partial derivative of the chemical source term in (5) implies that $\mathcal{R}_{j}$, the rate of progress of reaction $j$, obeys $\mathcal{R}_{j}\left(\xi_{j}\right)=\mathrm{UF}_{j}^{\xi_{j}} \mathcal{R}_{j}^{0}$, where $\mathcal{R}_{j}^{0}$ is the rate of progress for the nominal reaction parameters. In the present analysis, we assume that this interpretation of the uncertainty factors holds for all elementary reactions involved in the detailed mechanism.

Note that for reversible elementary reactions in which the reverse rate is specified using the reaction's equilibrium constant, the expression of the partial derivative of the chemical source term in (5) follows immediately from (3) and (4). This is also the case for irreversible reactions. For other reaction types, additional care must be exercised to ensure that (5) is indeed satisfied. Specifically, when Arrhenius parameters are specified for the reverse reaction rate, we assume that both the forward and backward pre-exponential parameters are perturbed by the germ in the same fashion. For pressure-dependent reactions, we also assume that the germ consistently perturbs all pre-exponential parameters. This assumption applies to pressure fall-off reactions, and pressure-dependent reactions using the PLOG formulation, as in both types multiple Arrhenius branches may be involved.

\subsection{Tangent linear approximation of the solution}

To estimate the sensitivity of the ignition delay time with respect to an infinitesimal germ perturbation, we rely on the linearized governing equation. Note that the linearization needs to be performed at individual realizations of the germ, $\boldsymbol{\xi}$, but we shall drop the explicit dependence of $\boldsymbol{\xi}$ to simplify the notation.

Let $\boldsymbol{Z}$ denote the state vector, concatenating the species mass fractions and temperature:

$$
Z_{i}= \begin{cases}Y_{i} & i=1, \ldots, N_{s} \\ T & i=N_{s+1}\end{cases}
$$

and let $\boldsymbol{Z}(t)$ denote the solution obtained at a given realization of the germ. Following a similar methodology as in [37], we estimate the local sensitivity of the state around $\boldsymbol{Z}(t)$, with respect to variations in $\xi_{j}$, $j=1, \ldots, N_{r}$, by integrating the linearized system:

$$
\frac{d \boldsymbol{Z}_{, j}}{d t}=J(t) \boldsymbol{Z}_{, j}+\boldsymbol{S}_{j} \quad j=1, \ldots, N_{r}
$$

with initial condition $\boldsymbol{Z}_{, j}(0)=0$. Here $\boldsymbol{Z}_{, j}(t) \equiv \partial \boldsymbol{Z} / \partial \xi_{j}$ denotes the partial derivative of the state at $\boldsymbol{Z}(t)$, $J(t)$ is the Jacobian of the right-hand side of (1) and (2) at $\boldsymbol{Z}(t), \boldsymbol{S}_{j}$ is the vector whose components, $S_{i j}$ are given by:

$$
S_{i j}(t)= \begin{cases}\frac{1}{\rho} q_{i}^{j}(t) & i=1, \ldots, N_{s} \\ -\frac{\gamma}{\rho c_{p}} \sum_{i=1}^{N_{s}} h_{i} q_{i}^{j}(t)+(\gamma-1) \frac{T W}{\rho} \sum_{i=1}^{N_{s}} \frac{1}{W_{i}} q_{i}^{j}(t) & i=N_{s}+1\end{cases}
$$

whereas $q_{i}^{j}(t)$ is the UF-scaled reaction source term at the unperturbed solution,

$$
q_{i}^{j}(t) \equiv \ln \left(\mathrm{UF}_{j}\right) \dot{\omega}_{i}^{j}(\boldsymbol{Z}(t)), \quad 1 \leq i \leq N_{s}+1, \quad 1 \leq j \leq N_{r} .
$$

Remark 3. It is advantageous to exploit the linearity of the system, namely by defining the scaled sensitivities

$$
\hat{\boldsymbol{Z}}_{, j} \equiv \frac{\boldsymbol{Z}_{, j}}{\ln \left(\mathrm{UF}_{j}\right)}, \quad j=1, \ldots, N_{r} .
$$


This scaling enables us to define the canonical system,

$$
\frac{d \hat{\boldsymbol{Z}}_{, j}}{d t}=J(t) \hat{\boldsymbol{Z}}_{, j}+\hat{\boldsymbol{S}}_{j}, \quad \hat{\boldsymbol{Z}}_{, j}(0)=0, \quad j=1, \ldots, N_{r},
$$

where $\hat{\boldsymbol{S}}_{j}$ is the vector with components $\hat{S}_{i j}$ given by:

$$
\hat{S}_{i j}(t)= \begin{cases}\frac{1}{\rho} \dot{\omega}_{i}^{j}(\boldsymbol{Z}(t)) & i=1, \ldots, N_{s} \\ -\frac{\gamma}{\rho c_{p}} \sum_{i=1}^{N_{s}} h_{i} \dot{\omega}_{i}^{j}(\boldsymbol{Z}(t))+(\gamma-1) \frac{T W}{\rho} \sum_{i=1}^{N_{s}} \frac{1}{W_{i}} \dot{\omega}_{i}^{j}(\boldsymbol{Z}(t)) & i=N_{s}+1\end{cases}
$$

The advantage of this system is that it is independent of the uncertainty factors, and depends only on elementary reaction rates of the unperturbed state. It is consequently convenient to integrate (14) and then recover the desired estimates of $\boldsymbol{Z}_{, j}$ using (13).

\subsection{Linearized approximation of ignition delay time sensitivity}

One of the challenges in estimating the local sensitivities of the ignition delay time is that $\tau$ is implicitly defined. Consequently, the local sensitivities of the state vector do not immediately yield the sensitivities of $\tau$. In this work, we rely on a linearized approximation to overcome this difficulty, specifically for the case where the ignition delay time is defined as the time argument at which the temperature of the mixture first reaches a specified threshold, i.e. $T(\tau)=T_{0}+\Delta T$, where $T_{0}$ is the initial mixture temperature, and $\Delta T$ is a user-specified temperature increase.

In the present context, we seek to estimate the time, $\tau+\delta \tau$, at which the perturbed temperature reaches the threshold $T_{0}+\Delta T$. In other words, we seek to estimate the increment $\delta \tau_{j}$ for a perturbation $\delta \xi_{j}$; we have:

$$
T(\tau+\delta \tau)+\frac{\delta T}{\delta \xi_{j}}(\tau+\delta \tau) \delta \xi_{j}=T_{0}+\Delta T .
$$

Linearizing the above equation, we get:

$$
\frac{d T}{d t}(\tau) \delta \tau+\frac{\delta T}{\delta \xi_{j}}(\tau) \delta \xi_{j} \approx 0
$$

which in the limit yields

$$
\frac{\partial \tau}{\partial \xi_{j}} \approx-\frac{\frac{\partial T}{\partial \xi_{j}}(\tau)}{\frac{d T}{d t}(\tau)}
$$

In the computational experiments below, we will verify the suitability of the linearized approximation by comparing the estimates (18) with finite-difference approximations based on independent (nonlinear) evaluations of the solution at perturbed values of the components of $\boldsymbol{\xi}$.

Remark 4. The gradient estimate in Eq. (18) leads to a sensitivity expression that is similar to Eq. (6) in [38] and identical to Eq. (10) in [40]. The present construction, however, differs slightly from the approach in [38]. Specifically, we arrived at Eq. (18) as a direct linearization of a functional of the solution, which enables us to offer the following perspective on its validity. To this end, we first note that for the linearization to be valid, the solution must remain differentiable with respect to the parameters throughout the time interval of interest; in particular, this rules out the occurrence of bifurcation phenomena. Second, for Eq. (18) to be useful, one must ensure that the temperature rate of change, $d T / d t(\tau)$, (i.e. the denominator) does not vanish. For exothermic systems, this condition is naturally met in a neighborhood of $\tau$, where $d T / d t$ achieves its peak value. 
Remark 5. In the development above, we have focused on the case where the uncertain rate parameters, $k_{j}$, follow log-uniform distributions. Of course, the methodology may be readily extended to other smooth distributions, e.g. when the rates are described in terms of Gaussian random variables. One should also note that the present methodology may be applied to other functionals of the solution, provided of course that these exhibit a smooth dependence on the parameters. Specifically, one may define the ignition delay time as the time needed by the system to reach a certain pressure, or a certain radical concentration. This would facilitate comparison with common experimental diagnostics such as pressure traces or $\mathrm{OH}$ concentration profiles. In section 4, we briefly illustrate such generalization for the case where $\tau$ is defined as the time needed to reach a certain pressure. In this case, one can follow a similar procedure as that outlined above to arrive at:

$$
\frac{\partial \tau}{\partial \xi_{j}} \approx-\frac{\frac{\partial P}{\partial \xi_{j}}(\tau)}{\frac{d P}{d t}(\tau)}
$$

where $P$ is the thermodynamic pressure. Differentiating the equation of state,

$$
P=\rho \Re\left(\sum_{i=1}^{N_{s}} \frac{Y_{i}}{W_{i}}\right) T,
$$

where $\Re$ is the universal gas constant, and using the fact that $\rho$ is independent of $t$ and of $\boldsymbol{\xi}$, this readily yields

$$
\frac{d P}{d t}(\tau)=\rho \Re T(\tau) \sum_{i=1}^{N_{s}} \frac{1}{W_{i}} \frac{d Y_{i}}{d t}(\tau)+\rho \Re\left(\sum_{i=1}^{N_{s}} \frac{Y_{i}(\tau)}{W_{i}}\right) \frac{d T}{d t}(\tau),
$$

and

$$
\frac{\partial P}{\partial \xi_{j}}(\tau)=\rho \Re T(\tau) \sum_{i=1}^{N_{s}} \frac{1}{W_{i}} \frac{\partial Y_{i}}{\partial \xi_{j}}(\tau)+\rho \Re\left(\sum_{i=1}^{N_{s}} \frac{Y_{i}(\tau)}{W_{i}}\right) \frac{\partial T}{\partial \xi_{j}}(\tau) .
$$

Substituting (21) and (22) into (19) we get:

$$
\frac{\partial \tau}{\partial \xi_{j}} \approx-\frac{T(\tau) \sum_{i=1}^{N_{s}} \frac{1}{W_{i}} \frac{\partial Y_{i}}{\partial \xi_{j}}(\tau)+\left(\sum_{i=1}^{N_{s}} \frac{Y_{i}(\tau)}{W_{i}}\right) \frac{\partial T}{\partial \xi_{j}}(\tau)}{T(\tau) \sum_{i=1}^{N_{s}} \frac{1}{W_{i}} \frac{d Y_{i}}{d t}(\tau)+\left(\sum_{i=1}^{N_{s}} \frac{Y_{i}(\tau)}{W_{i}}\right) \frac{d T}{d t}(\tau)},
$$

which may be readily applied once the state sensitivities are estimated.

Alternatively, the functional may be defined based on the fuel consumption, which would enable the methodology to be extended to isothermal systems. We finally note that the machinery may also be extended to other systems, such as oxidation at constant pressure, and to address sensitivities to other parameters, such as thermodynamic parameters. As mentioned earlier, this would be our focus in a follow up study.

\subsection{Balanced stretching eigenvector}

Note that when the germ, $\boldsymbol{\xi}$, is perturbed along the direction of the eigenvector $\boldsymbol{\eta}$ whose components are defined in (8), an analytical solution is available for the (nonlinearly) perturbed problem. Specifically, it is readily verified that once an unperturbed solution $\boldsymbol{Z}(t ; \boldsymbol{\xi})$ is determined, perturbed solutions for germs of the form $\boldsymbol{\xi}+\lambda \boldsymbol{\eta}$ with $\lambda \in \mathbb{R}$, can be obtained from

$$
\boldsymbol{Z}(\exp (\lambda) t ; \boldsymbol{\xi}+\lambda \boldsymbol{\eta})=\boldsymbol{Z}(t ; \boldsymbol{\xi})
$$


Consequently, if one considers vanishingly small perturbations along $\boldsymbol{\eta}$, one can readily exploit (24) to estimate the Gâteaux derivative,

$$
\boldsymbol{Z}_{, \boldsymbol{\eta}}(t) \equiv \nabla_{\boldsymbol{\xi}} \boldsymbol{Z}(t ; \boldsymbol{\xi}) \cdot \boldsymbol{\eta}=t \frac{d \boldsymbol{Z}(t ; \boldsymbol{\xi}))}{d t}
$$

In the numerical experiments below, we explore the possibility of exploiting the analytical relationships in (25) to correct results of the numerical integration of (10). Specifically, we consider an "amplitude correction" approach based on stretching the estimated values of the local derivatives so that (25) is satisfied. Letting $Z$ denote a generic component of the state vector $Z$, and $Z, j(t)$ the partial derivatives of $Z$ at time $t$, estimated by numerically integrating (10), or alternatively (14). The amplitude correction amounts to expressing the corrected vector according to

$$
Z_{, j}^{a c}(t)=\alpha Z_{, j}(t)
$$

and selecting $\alpha$ so that $Z_{, j}^{a c}$ satisfies (25). This leads to:

$$
\alpha=\frac{t \frac{d Z}{d t}}{\sum_{j} Z_{, j}(t) \eta_{j}}
$$

In section 4, we assess the suitability of this approach to obtain refined sensitivity estimates.

Remark 6 . From the transformation in (24) it readily follows that

$$
\tau(\boldsymbol{\xi}+\lambda \boldsymbol{\eta})=\exp (-\lambda) \tau(\boldsymbol{\xi}),
$$

which in turn leads to

$$
\tau_{, \boldsymbol{\eta}}(\boldsymbol{\xi})=\sum_{j=1}^{N_{r}} \frac{\partial \tau}{\partial \xi_{j}} \eta_{j}=-\tau(\boldsymbol{\xi}) .
$$

The analytical result above is in fact consistent with the linearized estimates of the ignition delay time sensitivities. To verify this claim, we simply evaluate the $\tau_{, \boldsymbol{\eta}}$ using the linearized estimates of the partial derivatives in (18). We get

$$
\begin{aligned}
& \tau_{, \boldsymbol{\eta}}=\sum_{j=1}^{N_{r}} \frac{\partial \tau}{\partial \xi_{j}} \eta_{j} \\
& \approx-\sum_{j=1}^{N_{r}} \frac{1}{\frac{d T}{d t}(\tau)} \frac{\partial T}{\partial \xi_{j}}(\tau) \eta_{j} \\
& =-\tau \text {, } \\
& \text { [using (25)] }
\end{aligned}
$$

which coincides with the result in (29). This establishes the claim.

Remark 7 . The analytical expression of the directional derivative, $\tau_{, \boldsymbol{\eta}}$ can also be used to obtain a relationship between the sensitivity indices in (6). Specifically, summing the sensitivity indices as expressed in (7), we have

$$
\sum_{j=1}^{N_{r}} S_{j}^{\tau}=\sum_{j=1}^{N_{r}} \eta_{j} \frac{1}{\tau} \frac{\partial \tau}{\partial \xi_{j}}=\frac{1}{\tau} \tau_{, \boldsymbol{\eta}}
$$

Substituting the result from (29) leads to

$$
\sum_{j=1}^{N_{r}} S_{j}^{\tau}=-1
$$

This identity can also be exploited to assess the accuracy of computational estimates obtained by integrating the linearized dynamics. 


\section{Numerical Scheme}

\subsection{Framework}

To assess different approaches for numerically integrating (10), we restrict ourselves to an "asynchronous" simulation framework in which the unperturbed numerical solution, $\boldsymbol{Z}(t)$, is computed in a preliminary stage, and recorded on fine time grid. Given that the solution $\boldsymbol{Z}(t)$ is computed using a stiff integration with an adaptive time step (see section 4), we assume that the state vector is stored on an irregular grid with step size $\Delta t_{i}>0, i=1, \ldots, N$, corresponding to a finite partition of the interval $[0, \tau]$. In other words, $\sum_{i=1}^{N} \Delta t_{i}=\tau$, and the partition has grid points specified by $t_{0}=0$, whereas

$$
t_{j}=\sum_{i=1}^{j} \Delta t_{i} \quad \text { for } 1 \leq j \leq N
$$

Because $\tau$ is a priori unknown, and because the solution changes slowly at early stages and rapidly as $t$ approaches $\tau$, it is generally impossible to specify a suitable time grid to record the solution before running the simulation. Practically, we overcome this hurdle by relying on a hybrid approach combining frequency-based and tolerance-based output strategies. The frequency-based strategy consists of recording the state using a fixed (predetermined) step size, whereas in the tolerance-based approach the solution is recorded whenever the temperature changes by a fixed threshold, which we fixed as $1 \mathrm{~K}$.

\subsection{Integration approach}

Using a given discrete history of the state vector, $Z^{n}, n=0, \ldots, N$, we explored different means of integrating (10) and consequently determining the partial derivatives on the corresponding grid, namely $Z_{, j}^{n}, j=1, \ldots, N_{r}, n=1, \ldots, N$, where the superscript denotes the time level. In our assessment below, we rely on a second-order implicit treatment based on the Crack-Nicolson scheme,

$$
\boldsymbol{Z}_{, j}^{n+1}=\left(\mathbb{I}-\frac{1}{2} \Delta t_{n+1} J^{n+1}\right)^{-1}\left[\boldsymbol{Z}_{, j}^{n}+\frac{1}{2} \Delta t_{n+1}\left(J^{n} \boldsymbol{Z}_{, j}^{n}+\boldsymbol{S}_{j}^{n+1}+\boldsymbol{S}_{j}^{n}\right)\right] \quad n=0, \ldots, N-1,
$$

where $\mathbb{I}$ is the $\left(N_{s}+1\right) \times\left(N_{s}+1\right)$ identity matrix.

\section{Results}

In this section, we assess the effectiveness of the methodology introduced in the previous sections in providing suitable estimates of local sensitivities of the ignition delay time to variations in the rate parameters. As outlined in section 2, attention is focused on variabilities specified in terms of uncertainty factors, affecting the rate of progress of elementary reactions. In this context, we consider mechanisms of different size, involving the oxidation of hydrogen (section 4.1), iso-octane (section 4.2), and n-butanol (section 4.3), in air, adiabatically and at constant volume.

The evolution of the state vector is simulated using TCHEM [36]. This library incorporates a stiff integration scheme [45, 46], with adaptive time stepping designed to maintain integration errors within user-specified tolerances. Because the ignition delay time is not known a priori, a sufficiently large simulation time is specified, so as to ensure that the ignition event occurs within the specified window. The output frequency is controlled by specifying two parameters (a) $\Delta t_{\max }$, which directly limits the largest time elapsed between consecutive writes, and (b) $\Delta T_{\max }$, which indirectly controls the output frequency by ensuring that the temperature change between two consecutive records of the state vector is less than $\Delta T_{\max }$. In other words, using the notations of section 3, the time difference $\Delta t_{i}$ between the output times $t_{i}$ and $t_{i-1}$ satisfies the following conditions: (a) $\Delta t_{i} \leq \Delta t_{\max }$, and (b) $\left|T_{i}-T_{i-1}\right| \leq \Delta T_{\max }$. Condition (b) is used in order to ensure that the rapid temperature rise during the ignition event is appropriately captured, without specifying 
tiny values of $\Delta t_{\max }$. This methodology results in a time history of the state vector $\boldsymbol{Z}$ on an irregular time grid, as considered in section 3 .

Our evaluation of TLA of ignition delay time sensitivities is conducted by post-processing the stored mixture state histories. This feature emphasizes the broad applicability of the approach, which can be applied to existing simulation databases, at the expense of potential efficiencies that may be gained by simultaneously simulating the evolution of the system and of its TLA.

Application of the TLA schemes outlined earlier is thus based on exploiting the available history of state vector, $\boldsymbol{Z}(t)$, to integrate system in (14). This task requires the evaluation of the Jacobian, $J$, and source terms $\hat{\boldsymbol{S}}_{j}$ defined in (15), corresponding to the instances of $\boldsymbol{Z}$ specified on the time grid. For this purpose, we rely on the Jacobian capabilities of TCHEM, and adapt the TCHEM chemical source term functions to compute the required source terms $\hat{\boldsymbol{S}}_{j}$. Finally, we rely on Eigen library [47] to perform matrix inversion and exponentiation.

Remark 8. As outlined in [36], TCHEM provides utility functions that estimate the Jacobian of the source either numerically or analytically. In the former case, a finite-difference methodology is used, using suitably small perturbations of the state vector needed to ensure accurate Jacobian estimates. In the latter case, analytical expressions are derived, applying as needed the chain rule to differentiate all terms appearing in the rate expressions. In the TCHEM release presently used, exact analytical expressions are available for all relevant reaction types, except for PLOG reactions. In this case, the analytical expressions are only approximate, as they ignore the pressure dependence of the Arrhenius parameters. Consequently, we explore the use of both the numerical and analytical Jacobian in all cases considered, with the understanding that the analytical Jacobian is approximate in the n-butanol case.

\section{1. $\mathrm{H}_{2}$ oxidation}

In this section, the TLA schemes are applied in a simple setting, in which we consider hydrogen oxidation in air at stoichiometric conditions $(\phi=1)$ with initial temperature, $T_{0}=1000 \mathrm{~K}$, and initial pressure, $p_{0}=1$ bar. The chemical mechanism from [48] is adopted for this purpose. This mechanism involves $N_{s}=9$ species, and $N_{r}=19$ reversible elementary reactions that do not exhibit pressure dependence. The computations are performed using TCHEM, with output frequency controlled by setting $\Delta T_{\max }=0.01 \mathrm{~K}$ and considering two different values of $\Delta t_{\max }$, namely $\Delta t_{\max }=10^{-7} \mathrm{~s}$ or $\Delta t_{\max }=10^{-6} \mathrm{~s}$. A time grid with 41834 intervals is obtained in the former case, whereas 40157 intervals are obtained in the latter.

We first assess the validity of the linearization approximation presented in the right-hand side of Eq. 18 . Hence, we plot in Fig. 1 the partial derivative $\partial \tau / \partial \xi_{j}$ versus reaction index $j$, contrasting estimates obtained using finite differences and using the linearized approximation. As can be seen in Fig. 1, the results are in excellent agreement thus indicating that, inthis example, the linearized approximation yields accurate estimates of the local sensitivities of ignition delay time. Specifically, the rms deviation between the local sensitivity vectors, normalized by the norm of the finite-difference vector, is $7.61 \times 10^{-6}$.

Next, we exploit the TLA schemes to estimate $\partial T / \partial \xi_{j}$, and consequently determine the local sensitivity indices $S_{j}^{\tau}$, for $j=1, \ldots, N_{r}$. Figure 2 shows $\partial T / \partial \xi_{j}$ versus reaction index $j$. Plotted are TLA results obtained using the analytical Jacobian before and after correction, as well as predictions obtained using the numerical Jacobian. In all cases, a time grid obtained using $\Delta t_{\max }=10^{-6} \mathrm{~s}$ are used. As can be seen, the TLA results obtained using the analytical and numerical Jacobian are in close agreement. More importantly, the predictions are also in close agreement with the brute-force finite-difference estimates.

Local sensitivity results obtained using TLA are analyzed in Fig. 3. Plotted are predictions obtained using $\Delta t_{\max }=10^{-6} \mathrm{~s}$ before and after correction, and $\Delta t_{\max }=10^{-7} \mathrm{~s}$ without correction. Consistent with the results obtained for $\partial T / \partial \xi_{j}$, the sensitivity predictions are in close agreement with finite-difference results. In addition, for the present case the TLA results are weakly sensitive to $\Delta t_{\max }$.

A quantitative analysis of the TLA errors is conducted by contrasting the TLA estimates to the finite difference results. Specifically, letting $g_{j}$ denote the TLA estimates of $T_{, j}$ or $S_{, j}$, and $\hat{g}_{j}$ their finite-difference 


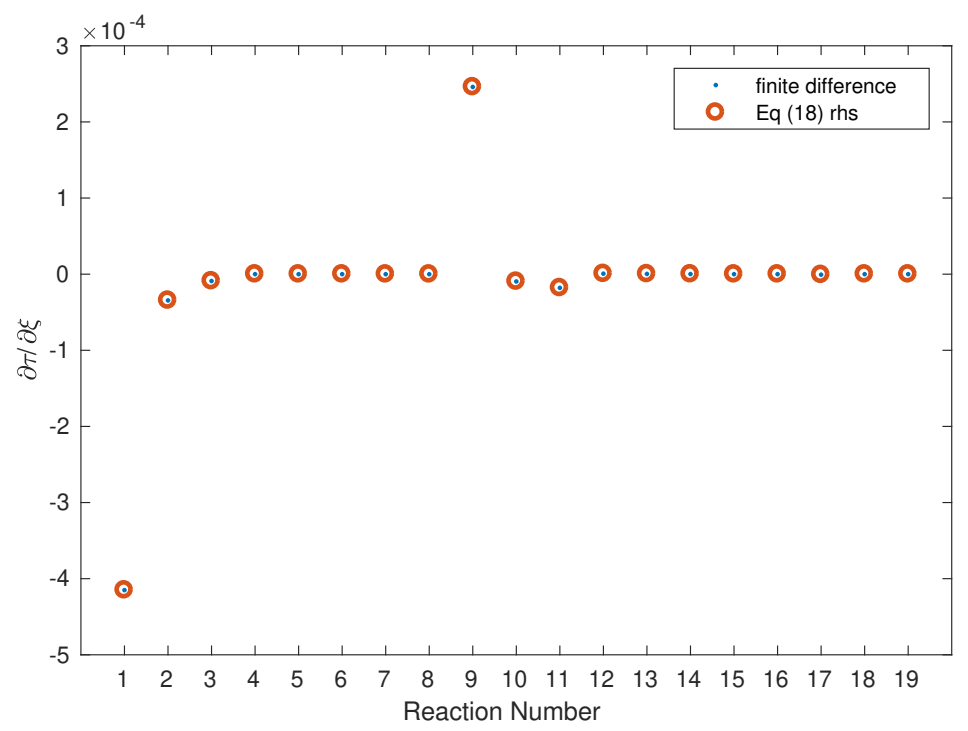

Figure 1: $\partial \tau / \partial \xi_{j}$ (s) versus reaction index $j$ for $\mathrm{H}_{2}$ oxidation. Plotted are estimates obtained using finite differences (blue dots) and the linearized approximation in Eq. 18 (orange circles).

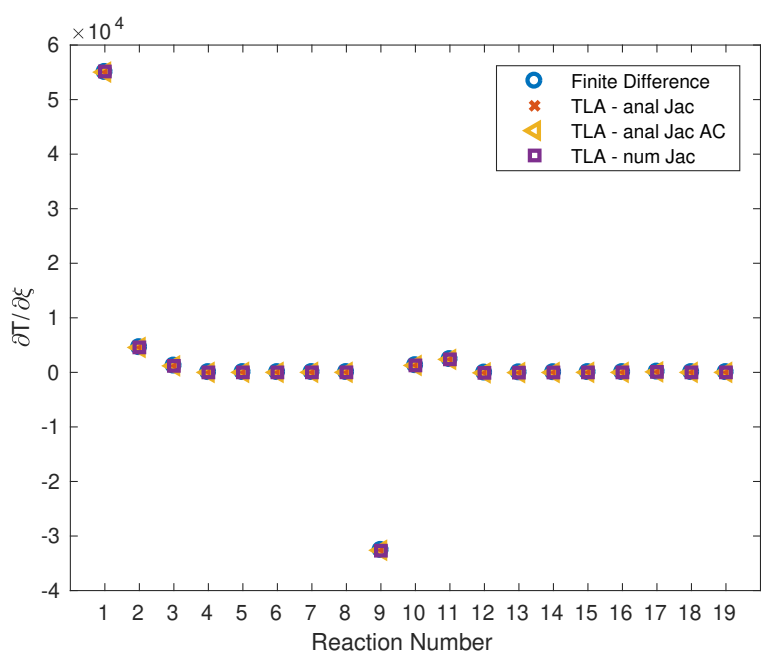

Figure 2: $\partial T / \partial \xi_{j}(\mathrm{~K})$ versus reaction index $j$ for $\mathrm{H}_{2}$ oxidation. Shown are results obtained using finite differences and TLA. For the analytical Jacobian case, the corrected predictions are also shown.

counterparts, we first estimate the root mean square (rms) error,

$$
E=\left(\frac{1}{N_{r}} \sum_{j=1}^{N_{r}}\left(g_{j}-\hat{g}_{j}\right)^{2}\right)^{1 / 2}
$$

and using the finite-difference results as reference to define the normalized errors,

$$
\mathcal{E}_{2}=\frac{\mathrm{E}}{\|\hat{\boldsymbol{g}}\|_{2}} \quad \text { and } \quad \bar{E}=\frac{\mathrm{E}}{\max _{i}\left|\hat{\mathrm{g}}_{i}\right|},
$$

where $\|\hat{\boldsymbol{g}}\|_{2}$ denotes the $\mathrm{L}_{2}$-norm of the vector $\hat{\boldsymbol{g}}$ having components $\hat{g}_{j}$. 


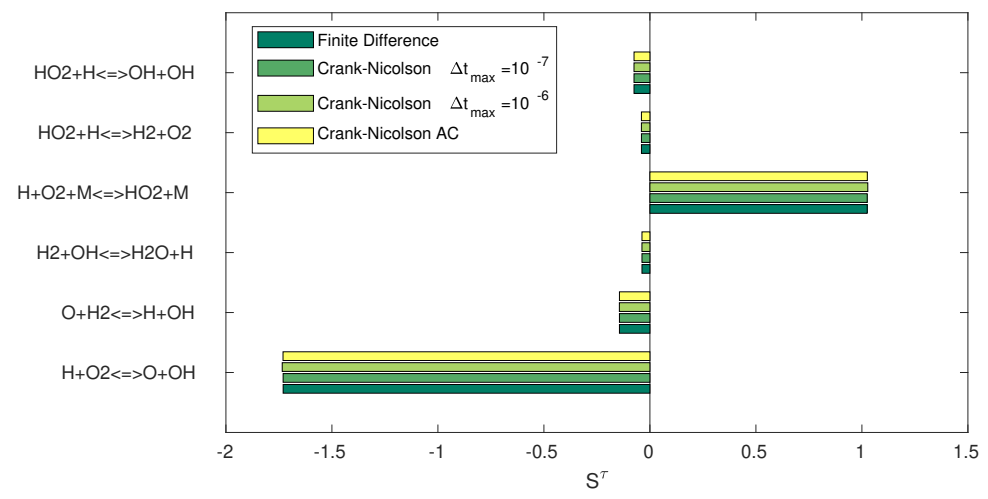

Figure 3: Sensitivity indices, $\mathrm{S}^{\tau}$, of the dominant reactions in the $\mathrm{H}_{2}$ oxidation case (a cutoff of 0.0375 was used). Plotted are results obtained using finite differences, and TLA approximations using the analytical Jacobian, and time grids obtained with $\Delta t_{\max }=10^{-7} \mathrm{~s}$ and $\Delta t_{\max }=10^{-6} \mathrm{~s}$. For the coarser time grid, the corrected predictions are also shown.

Table 1 shows the normalized errors for the TLA estimates of $T_{, j}$. We report normalized errors computed before and after amplitude correction. For the fine time grid, the errors are very small, indicating that accurate predictions are obtained with the TLA methodology. The normalized errors are larger when integration is performed on the coarser grid, but the normalized errors drop appreciably when the amplitude correction scheme is applied. Also note that in the present case the estimates obtained using analytical and numerical Jacobians are essentially identical, as anticipated. In addition, the computed normalized rms error for $T_{, j}$ and $S_{, j}$ (not shown) are nearly identical. This is not surprising because the linearized approximation of the ignition delay sensitivity is highly accurate, so that errors in the TLA approximation of the ignition delay time sensitivities $\left(S_{, j}\right)$ are dominated by the integration errors occurring in the computation of the local sensitivities of temperature $\left(T_{, j}\right)$.

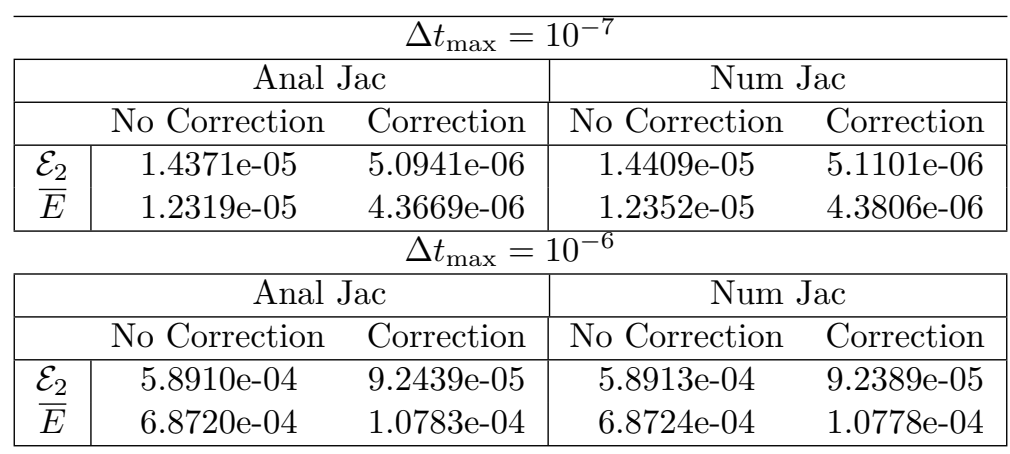

Table 1: Normalized rms error for $T_{, j}$ for the $\mathrm{H}_{2}$ oxidation case. Top table: integration is performed a time grid obtained with $\Delta t_{\max }=10^{-7} \mathrm{~s}$. Bottom table: integration is performed on time grid obtained with $\Delta t_{\max }=10^{-6} \mathrm{~s}$. Errors estimated before and after amplitude correction are reported, using results obtained with the analytical and numerical Jacobians.

We have also explored the possibility of defining the ignition delay time using the history of the pressure signal. To facilitate comparison with the temperature-based analysis, we have defined the ignition pressure to be value at which the mixture temperature reaches $T_{0}+400 \mathrm{~K}$. This ensures that the ignition delay time in both approaches is identical. We have assessed the sensitivities $\partial \tau / \partial \xi_{j}$ using (i) the brute-force finite-difference approach, (ii) the linearized approximation using the independent realizations involved in the finite-difference analysis, as well as (iii) the TLA-based state sensitivities in conjunction with (23). Results from all three approaches (not shown) were in excellent agreement with each other, and essentially indistinguishable from the temperature-based results depicted in Fig. 1. This illustrates the flexibility of 
using TLA-based sensitivities in a broad class of linearized solution functionals.

\subsection{Iso-octane oxidation}

In this section, we consider a more elaborate example consisting of the combustion of a stoichiometric $(\phi=1)$ iso-octane air mixture, adiabatically at constant volume, starting from an initial temperature $T_{0}=850 \mathrm{~K}$ and an initial pressure $p_{0}=20$ bar. The combustion of iso-octane is described using the detailed chemical mechanism from [49-51], which involves $N_{s}=874$ species, and $N_{r}=3796$ elementary reactions. The reactions include both reversible and irreversible reactions, as well as pressure-dependent reactions. The computations are performed using TCHEM, with output frequency controlled using $\Delta T_{\max }=1 \mathrm{~K}$ and $\Delta t_{\max }=10^{-5} \mathrm{~s}$. This yields a discrete sequence of state vectors specified at the boundaries of 2569 irregularly spaced sub-intervals, forming a partition of the interval $[0, \tau]$. The (nominal) ignition delay time is computed to be $\tau=0.0231 \mathrm{~s}$.

As in the case of $\mathrm{H}_{2}$ above, we start by assessing the validity of the linearized approximation of the ignition delay time sensitivities. To this end, we contrast in Fig. 4 the estimates of $\partial \tau / \partial \xi_{j}$ for the finite difference approach and the linearized approximation. The figure depicts the results for indices $j$ selected according to the finite difference results' highest absolute values. The normalized rms difference between the two full vectors of sensitivities is $7.7 \times 10^{-5}$. The small relative rms value provides confidence in the quality of the linearized approximation of $\partial \tau / \partial \xi_{j}$.

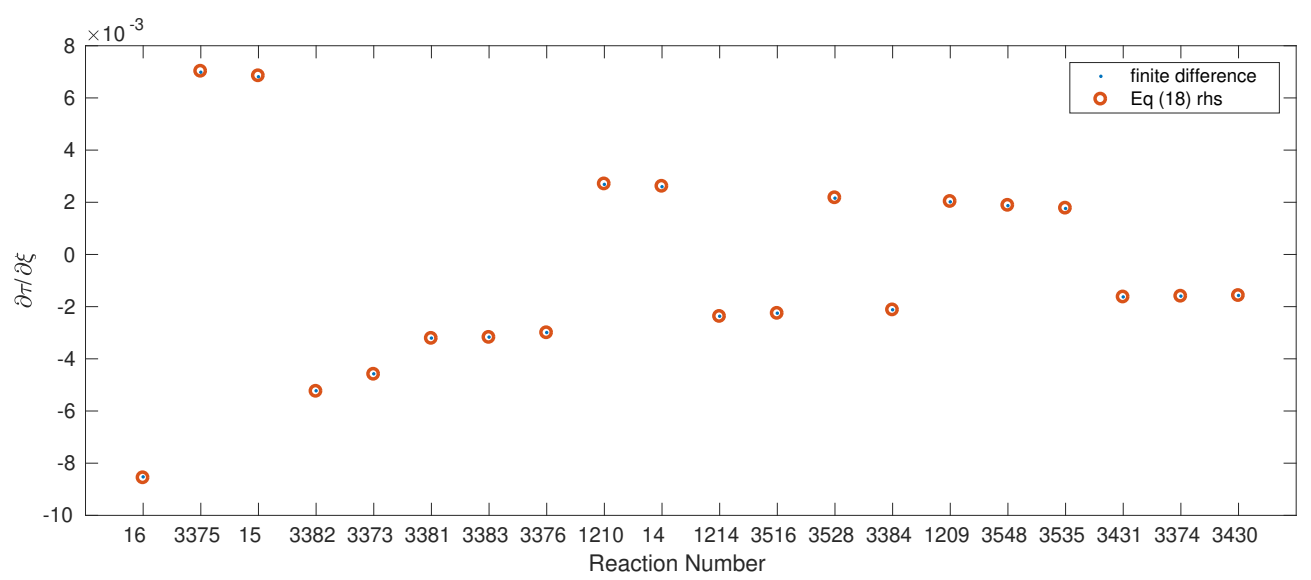

Figure 4: $\partial \tau / \partial \xi_{j}(\mathrm{~s})$ versus reaction index $j$ for iso-octane oxidation. Plotted are estimates obtained using finite differences (blue dots) and the linearized approximation, Eq. 18 (orange circles). The reaction indices shown correspond to the largest absolute values of the finite difference estimates of $\partial \tau / \partial \xi_{j}$, and are sorted in descending order.

Figures 5 and 6 predictions of the TLA approximation of $T_{j}$ and $S_{, j}$, respectively. Plotted in Fig. 5 are the TLA predictions of $T_{j}$, for the same indices $j$ selected in Fig. 4. Finite-difference results are plotted for comparison, and corrected predictions for the analytical Jacobian case are also depicted. As can be seen from Fig. 6, the correction scheme may lead to appreciable refinement of the TLA integration results. Similarly, Fig. 6 contrasts finite-difference results with the corresponding TLA estimates. The results indicate that the TLA predictions are in very good agreement with finite-difference results.

A quantitative assessment of TLA approximation errors was conducted. Specifically, normalized rms errors are estimated using Eq. (33) for the $N_{r}$-dimensional sensitivities $T_{, j}$ and $S_{, j}$. As in the case $H_{2}$, the results for $T_{, j}$ and $S_{, j}$ are nearly identical, and so only results for $T_{, j}$ are reported in Table 2 . As can be seen, the normalized errors are very small in all cases. Thus, the TLA is effective for this detailed mechanism involving hundreds of species and thousands of elementary reactions.

\section{3. n-butanol oxidation}

In this section, we consider combustion of a stoichiometric n-butanol air mixture with initial temperature, $T_{0}=800 \mathrm{~K}$, and initial pressure, $p_{0}=40$ bar. We rely on the n-butanol mechanism of Sarathy et al. [52], 


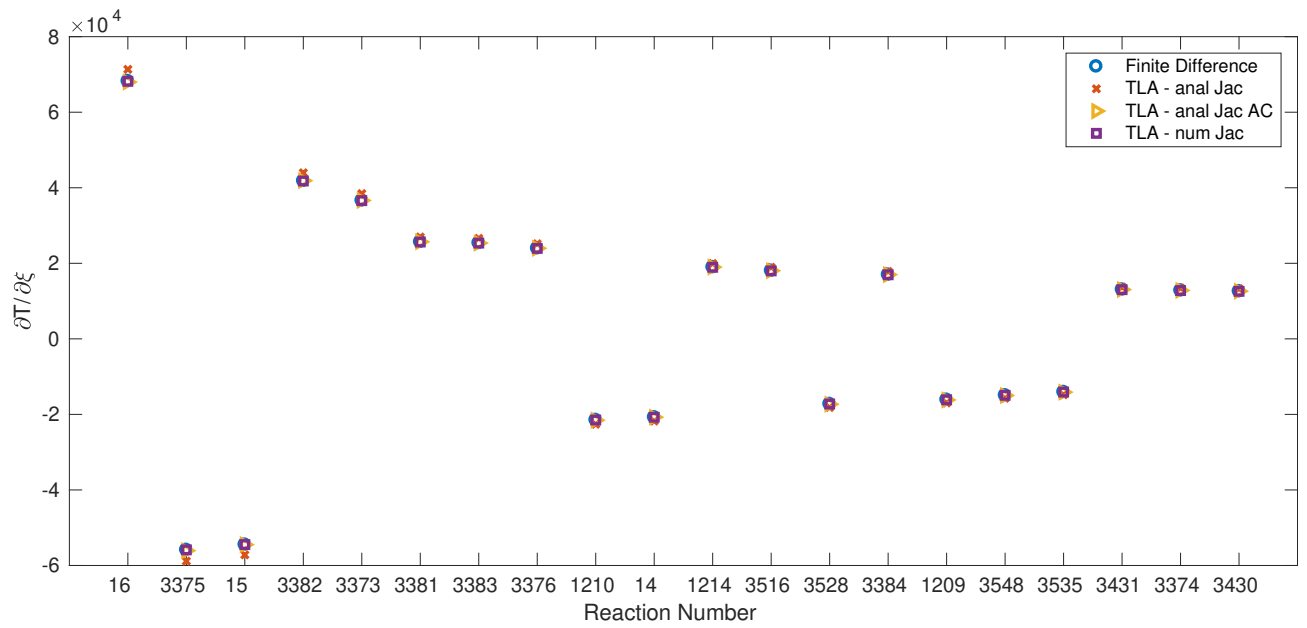

Figure 5: $\partial T / \partial \xi_{j}(\mathrm{~K})$ versus reaction index $j$ for iso-octane oxidation. Shown are results obtained using finite differences, and TLA using analytical Jacobian (before and after correction) and numerical Jacobian (without correction). The integration is performed on time grid obtained with $\Delta t_{\max }=10^{-5} \mathrm{~s}$, comprising 2569 intervals.

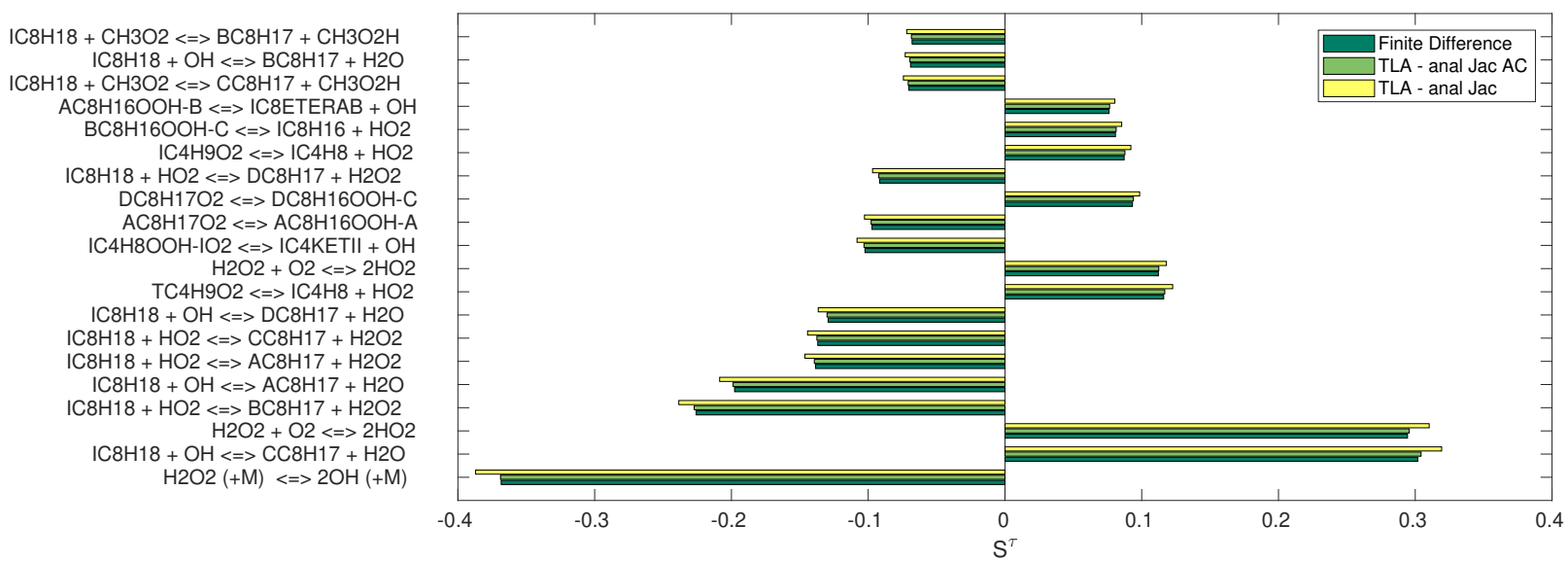

Figure 6: Sensitivity indices, $\mathrm{S}^{\tau}$, for individual reactions in the iso-octane oxidation case. Plotted are results obtained using finite differences, and TLA approximations shown in Fig. 5. The reactions are arranged from bottom to top using the same sequence of reaction indices shown on the axis of Fig. 4.

\begin{tabular}{|c|cc|cc|}
\hline \multicolumn{3}{|c|}{ Anal Jac } & \multicolumn{2}{c|}{ Num Jac } \\
\hline \multicolumn{2}{|c|}{ No Correction } & Correction & No Correction & Correction \\
\hline $\mathcal{E}_{2}$ & $8.1067 \mathrm{e}-04$ & $8.6531 \mathrm{e}-05$ & $7.6540 \mathrm{e}-05$ & $7.8317 \mathrm{e}-05$ \\
$\bar{E}$ & $1.7780 \mathrm{e}-03$ & $1.8979 \mathrm{e}-04$ & $1.6787 \mathrm{e}-04$ & $1.7177 \mathrm{e}-04$ \\
\hline
\end{tabular}

Table 2: Normalized rms error for $T_{, j}$ for the iso-octane oxidation case. Error estimates obtained before and after amplitude correction are reported using analytical and numerical Jacobians.

which involves $N_{s}=687$ species and $N_{r}=3435$ reactions. For the finite-difference sensitivities, we restrict our attention to reactions involved in the rate rules, i.e. perturbations in the rates of elementary reactions appearing in the "base mechanism" are ignored. This simplification leads us to consider 30 reaction classes [7], comprising a total of $N_{r}^{\prime}=518$ reactions that involve higher hydrocarbons $\left(\mathrm{C}_{5}\right.$ and larger $)$. Various reaction types involved in the detailed n-butanol mechanism, including reversible, irreversible, pressure falloff, and 
pressure-dependent reaction using the PLOG formulation. Note, however, that when the TLA schemes are applied, we may readily consider the local sensitivities of all reactions, because the cost of the integration is primarily dominated by Jacobian inversion. To perform the integration, we rely on the history of state vectors obtained from TCHEM simulations, performed by setting $\Delta T_{\max }=1 \mathrm{~K}$, and $\Delta t_{\max }=10^{-6} \mathrm{~s}$. Ignition occurs at $\tau=0.0052 \mathrm{~s}$. With the selected output control parameters, the discrete states are recorded at the nodes of an irregular time grid having 5366 intervals.

Figure 7 shows the estimates of $\partial \tau / \partial \xi_{j}$ as obtained from finite differences and from the linearized approximation. As in the previous examples, the results are in excellent agreement, highlighting the accuracy of the TLA estimates. In Fig. 8, we present estimates of $\partial T / \partial \xi_{j}$ using finite differences and TLA; plotted are results obtained using the approximate analytical Jacobian (with and without correction) and the numerical Jacobian. As can be seen, the uncorrected results obtained using the numerical Jacobian are in close agreement with the finite-difference results, but the corresponding predictions obtained using the approximate analytical Jacobian exhibit significant discrepancies. This is not surprising because, in this case, the analytical expressions used are only approximate. Note, however, that when the correction scheme is applied the analytical Jacobian results are once again consistent with the finite-difference predictions. Thus, the correction scheme enables us to overcome amplitude errors resulting from the Jacobian approximation. Similar trends are observed in the corresponding sensitivity results reported in Fig. 9.

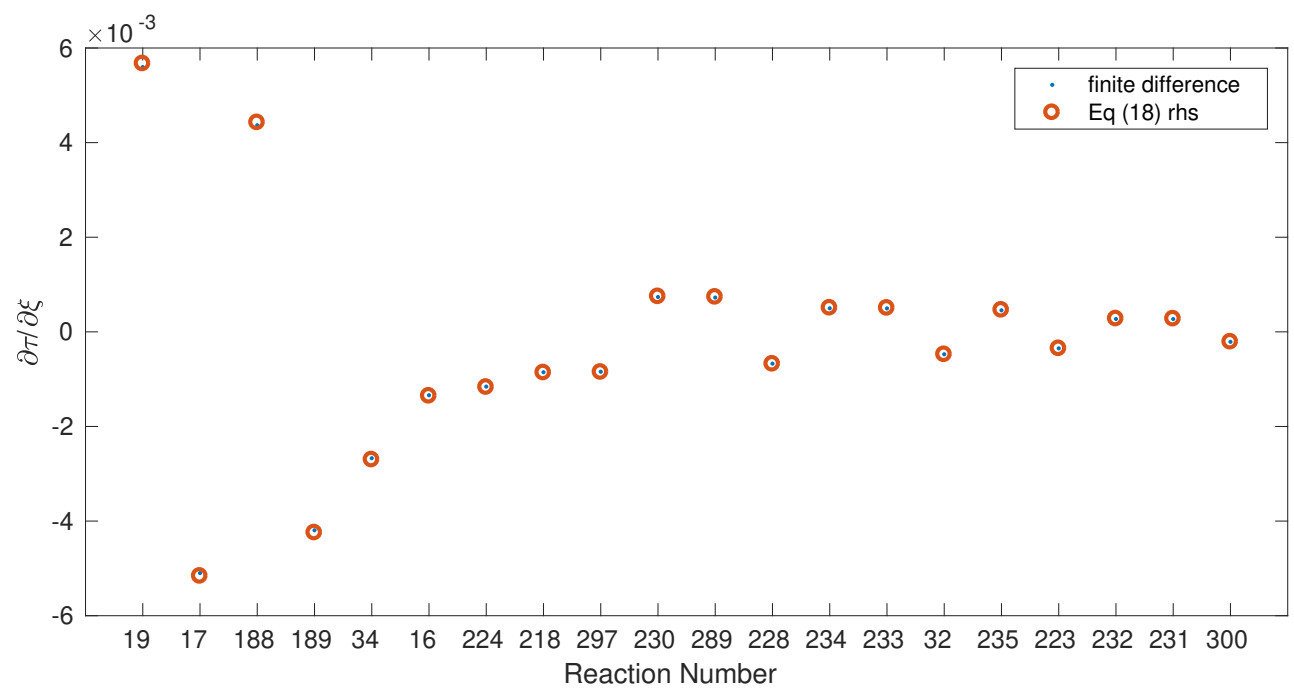

Figure 7: $\partial \tau / \partial \xi_{j}(\mathrm{~s})$ versus reaction index $j$ for n-butanol oxidation. Plotted are estimates obtained using finite differences (blue dots) and the linearized approximation, Eq. 18 (orange circles). The reaction indices shown correspond to the largest absolute values of the finite difference estimates of $\partial \tau / \partial \xi_{j}$, and are sorted in descending order. The normalized rms deviation between the local sensitivity vectors is $5.56 \mathrm{e}-04$.

In the order to preform a quantitative error analysis, we adapt the definition of the rms error as follows:

$$
E^{\prime}=\left(\frac{1}{N_{r}^{\prime}} \sum_{j=1}^{N_{r}^{\prime}}\left(g_{j}-\hat{g}_{j}\right)^{2}\right)^{1 / 2}
$$

where the summation is performed over the $N_{r}^{\prime}=518$ indices $j$ of the reactions belonging to the 30 reaction classes. As discussed earlier, the vector $g_{j}$ is used to denote the local sensitivities $T_{, j}$ or $S_{, j}$ determined using the TLA, whereas $\hat{g}_{j}$ is used to denote the corresponding finite-difference estimates. Using the finitedifference results as reference, we define the normalized errors

$$
\mathcal{E}_{2}^{\prime}=\frac{\mathrm{E}^{\prime}}{\|\hat{\boldsymbol{g}}\|_{2}} \quad \text { and } \quad \overline{E^{\prime}}=\frac{\mathrm{E}^{\prime}}{\max _{j}\left|\hat{\mathrm{g}}_{j}\right|},
$$




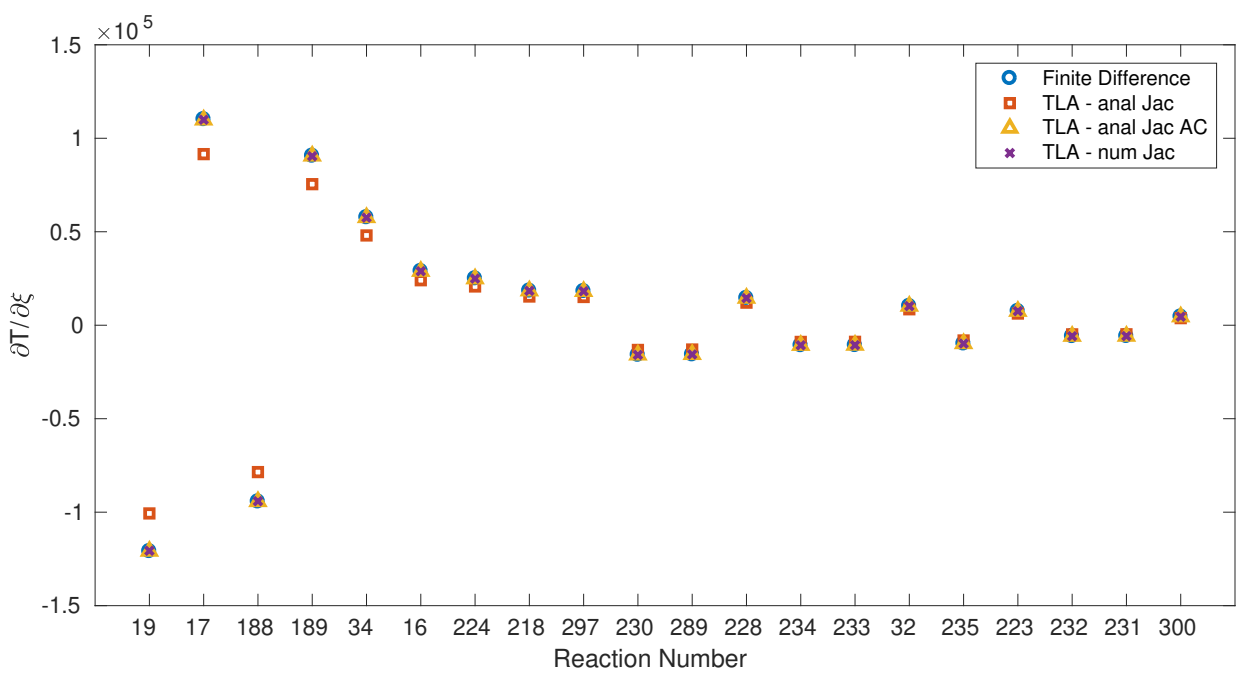

Figure 8: $\partial T / \partial \xi_{j}(\mathrm{~K})$ versus reaction index $j$ for n-butanol oxidation. Shown are results obtained using finite differences, and TLA approximations as indicated. The integration is performed on time grid obtained with $\Delta t_{\max }=10^{-6} \mathrm{~s}$, comprising 5366 intervals.
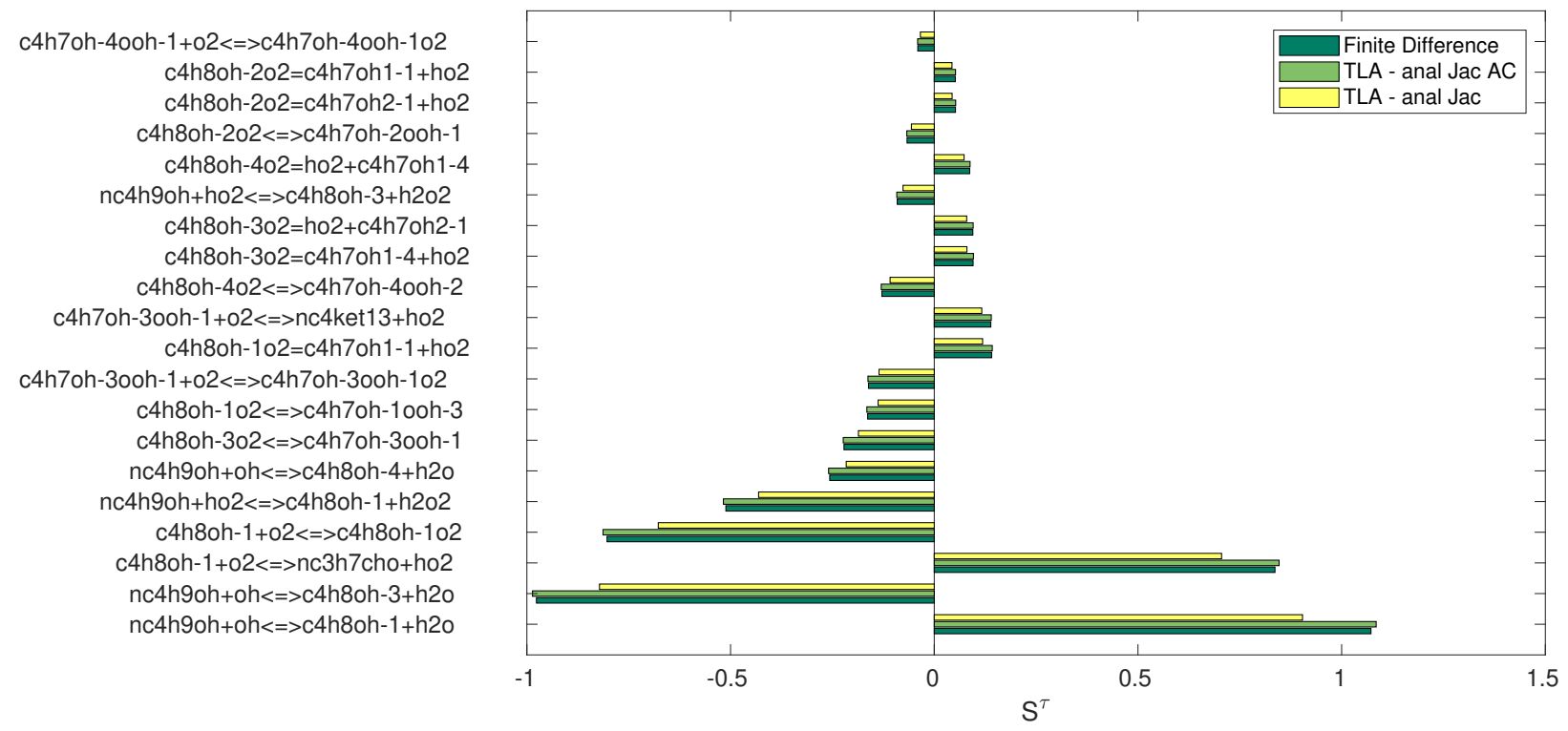

Figure 9: Sensitivity indices, $\mathrm{S}^{\tau}$, for individual reactions in the n-butanol oxidation case. Plotted are results obtained using finite differences, and TLA approximations as indicated. The reactions are arranged from bottom to top using the same sequence of reaction indices shown on the axis of Fig. 7 .

where $\|\hat{\boldsymbol{g}}\|_{2}$ denotes the $\mathrm{L}_{2}$-norm of the $N_{r}^{\prime}$-dimensional vector $\hat{\boldsymbol{g}}$ having components $\hat{g}_{j}$.

The normalized rms errors defined by (35) are reported in Table 3. Similar to $\mathrm{H}_{2}$ and iso-octane cases, the results show very small errors.

\subsection{Performance}

This section analyzes the computational efficiency of the TLA approach by contrasting its performance with the finite-difference approach. 


\begin{tabular}{|c|cc|cc|}
\hline \multicolumn{2}{|c|}{ Anal Jac } & \multicolumn{2}{c|}{ Num Jac } \\
\hline & No Correction & Correction & No Correction & Correction \\
\hline $\mathcal{E}_{2}$ & $7.3740 \mathrm{e}-03$ & $7.6611 \mathrm{e}-05$ & $9.6957 \mathrm{e}-05$ & $1.1328 \mathrm{e}-04$ \\
$E$ & $1.3746 \mathrm{e}-02$ & $1.4282 \mathrm{e}-04$ & $1.8075 \mathrm{e}-04$ & $2.1118 \mathrm{e}-04$ \\
\hline
\end{tabular}

Table 3: Normalized rms error for $T_{, j}$ in the n-butanol oxidation case. Results are shown for STLA based on Crank-Nicolson $(\mathrm{CN})$ integration. Error estimates obtained before and after amplitude correction are reported using both analytical and numerical Jacobians.

Because the finite-difference approach is based on perturbing rates individually for each elementary reaction and performing a forward simulation for each perturbation, the overall cost incurred essentially amounts to the cost of a single simulation multiplied by the total number of reaction sensitivities sought. Thus, if for each mechanism one uses the cost of a single simulation as a normalizing factor, then the normalized CPU time required by the finite-difference approach is $N_{r}$ for the $\mathrm{H}_{2}$ and iso-octane examples. On the other hand, the normalized CPU cost in the n-butanol example is $N_{r}^{\prime}$, because the rate perturbations were restricted to reactions associated with the rate rules.

\begin{tabular}{|c|c|c|c|c|}
\hline \multicolumn{3}{|c|}{$\mathrm{H}_{2}$} & n-butanol & iso-octane \\
\hline$\Delta t_{\max }$ & $10^{-6}$ & $10^{-7}$ & $10^{-6}$ & $10^{-5}$ \\
\hline $\mathrm{FL}$ & 1 & 15 & 518 & 3796 \\
\hline Anal Jac & 1.97 & 2.5 & 5.5 & 2.52 \\
\hline Num Jac & 1.64 & 2.075 & 4.92 & 2.09 \\
\hline
\end{tabular}

Table 4: Computational cost of various approaches, normalized by the CPU time of a single forward simulation. Reported are CPU cost the finite-difference (FD) approach, of the TLA using analytical and numerical Jacobians.

Normalizing the CPU cost incurred in the TLA applications in the same manner as in finite differences, we can readily assess the performance of the TLA. As can be seen from Table 4, in the $\mathrm{H}_{2}$ case a remarkable speed-up is achieved by the TLA, which requires a CPU more than 7 times smaller than the finite-difference approach. In the iso-octane and n-butanol examples involving large detailed mechanisms, the TLA methods are faster by an order of magnitude. These savings occur even when the finite-difference perturbations are restricted to reaction classes, as in the n-butanol example.

\subsection{Discussion}

We conclude with a brief discussion of the fidelity of the linearized approximation of the ignition delay time as provided in (18) and of the resulting sensitivity estimates obtained via TLA, as well as the performance of the TLA scheme.

In the present analysis, we have performed a step-wise error analysis, in which we first tested Eq. (18) using non-linear estimates obtained using the brute-force approach. Briefly, our experiences in all cases considered point to the validity of Eq. (18) as insignificant discrepancies were found. This inherently relied on care exercised in (i) accurately estimating the solution, namely by selecting sufficiently small tolerances for the stiff integration, (ii) using a time grid that is sufficiently fine to minimize numerical errors in estimating $\tau[38]$, which is presently achieved by interpolating between grid points that bracket the selected temperature value, and (iii) selection of very small perturbation parameters, so that accurate finite-difference estimates can be consequently obtained [53].

Concerning the fidelity of the TLA sensitivity estimates, our experiences indicate that very small error levels can indeed be achieved, appreciably smaller than those reported in [38] and [40] using the direct differentiation approach. Similar to experiences obtained with alternatives afforded by BVP [40] and adjoint [39] methodology, it appears that TLA and direct sensitivity results can be obtained that are sharp enough to enable robust determination of active subspaces. This will be explored in follow up work. 
Concerning performance, it was noted in [38] that the direct approach would lead to speed up factors that are proportional to the number of reactions in the chemical mechanism, though actual performance estimates are not reported. This implies an $\mathrm{O}(1)$ complexity, with actual costs that are approximately equal to or smaller than the cost of a single forward simulation. Though not explored in the present simulations, this may be achieved through an optimization exercise based on saving the state, the source term, the Jacobian and its factorization on a suitable time grid as they are computed in the forward calculation, or alternatively integrating the original system and direct sensitivity equations simultaneously as suggested in $[37]$.

Finally, we contrast the present performance estimates with those reported in [39] using the adjoint methodology, and in [40] using BVP. Specifically, for the GRI3.0 mechanism $\left(N_{r}=325\right)$, speed up factors of about 2-3 were reported in [39], which can be further improved to exceed one order of magnitude. For the Aramco mechanism $\left(N_{r}=2716\right)$ reported CPU times in [40] imply a speed up factor of about 43 . Our present performance experiences compare favorably with those reported in [39] and [40]. In particular, for the iso-octane mechanism $\left(N_{r}=3796\right)$, speed up factors for the TLA exceeding 1000 are achieved.

\section{Conclusions}

A tangent linear approximation is developed to estimate the local sensitivity of the ignition delay time with respect to uncertainty in individual rate parameters. Attention is focused on a gas mixture reacting adiabatically at constant volume. The uncertainty in the rates of elementary reactions is described by uncertainty factors, and parametrized using independent canonical random variables. Specifically, the formulation assumes a log-uniform dependence of the rate of progress of individual reactions on the associated random variables.

The framework developed is based on a combination of two key ingredients. The first consists in deriving of a canonical tangent linear system governing the evolution of the sensitivities of the state vector to individual components of the germ. The second consists in developing of a linearized approximation that relates the ignition delay time sensitivity to the sensitivities of the state vector.

The proposed framework was evaluated by applying the machinery in a post-processing mode, using as input a discrete sequence of state vectors specified on an irregular time mesh. With this information, the tangent linear system is integrated in time, yielding the sensitivities the state vector. An amplitude correction strategy was also investigated, capitalizing on an analytical transformation that holds for perturbations taken defined along the balanced stretching eigenvector.

The effectiveness of the proposed methodology was assessed based on simulations of the combustion of hydrogen, n-butanol, and iso-octane in air at intermediate pressures and temperatures. For the purpose of verifying the results and quantifying performance gains, a brute-force sensitivity analysis was applied, based on perturbing the rates of individual reactions one at a time, and conducting independent ignition simulations for each of the perturbed rates. In particular, the analysis of simulation results revealed that:

1. For all cases considered, the linearized approximation of the local sensitivity of the ignition delay time led to results that are in very close agreement with finite-difference estimates. This provides a well-established foundation for local sensitivities obtained via TLA.

2. For all mechanisms considered, the TLA results obtained were in good agreement with finite-difference results. Furthermore, TLA estimates could be improved through the application of an amplitude correction scheme. This provides an effective means of obtaining robust sensitivity estimates, even when derived from a sequence of state vectors specified on relatively coarse, irregular time grids.

3. The performance analysis showed that for the relatively small $\mathrm{H}_{2}$ oxidation mechanism, the TLA is computationally competitive with the finite-difference approach. For large detailed mechanisms, the TLA can offer order-of-magnitude enhancement in computational efficiency, even when finite-difference perturbations are restricted to a subset of reactions associated with rate rules. Specifically, the TLA achieves a speed-up factor of 7.6 for the case of $\mathrm{H}_{2}$ ignition, 93 for the n-butanol mechanism, and 1506 for iso-octane. 
In future work, we plan to consider the extension of the present TLA framework to address local sensitivity of the ignition delay time due to variation in thermodynamic parameters. We also plan to rely on the resulting capabilities to explore the application of active subspace methodologies in high dimension.

\section{Acknowledgments}

The research reported in this publication was supported by King Abdullah University of Science and Technology (KAUST). The authors are grateful to three anonymous reviewers for comments and suggestions that resulted in significant improvements to this manuscript. The TLA codes are available from the authors upon request.

\section{References}

[1] M. Fikri, J. Herzler, R. Starke, C. Schulz, P. Roth, G. Kalghatgi, Autoignition of gasoline surrogates mixtures at intermediate temperatures and high pressures, Combustion and Flame 152 (2008) 276-281.

[2] S. S. Goldsborough, A chemical kinetically based ignition delay correlation for iso-octane covering a wide range of conditions including the NTC region, Combustion and Flame 156 (2009) 1248-1262.

[3] X. He, M. Donovan, B. Zigler, T. Palmer, S. Walton, M. Wooldridge, A. Atreya, An experimental and modeling study of iso-octane ignition delay times under homogeneous charge compression ignition conditions, Combustion and Flame 142 (2005) 266-275.

[4] K. Heufer, R. Fernandes, H. Olivier, J. Beeckmann, O. Röhl, N. Peters, Shock tube investigations of ignition delays of n-butanol at elevated pressures between 770 and 1250K, Proc. Combust. Inst. 33 (2011) 359-366.

[5] I. Stranic, D. P. Chase, J. T. Harmon, S. Yang, D. F. Davidson, R. K. Hanson, Shock tube measurements of ignition delay times for the butanol isomers, Combust. Flame 159 (2012) 516-527.

[6] B. W. Weber, K. Kumar, Y. Zhang, C.-J. Sung, Autoignition of n-butanol at elevated pressure and low-to-intermediate temperature, Combust. Flame 158 (2011) 809-819.

[7] M. Hantouche, S. Sarathy, O. Knio, Global sensitivity analysis of n-butanol reaction kinetics using rate rules, Combust. Flame 96 (2018) 452-465.

[8] M. Reagan, H. Najm, B. Debusschere, O. L. Maître, O. Knio, R. Ghanem, Spectral stochastic uncertainty quantification in chemical systems, Combust. Theory Model. 8 (2004) 607-632.

[9] M. T. Reagan, H. Najm, P. Pebay, O. Knio, R. Ghanem, Quantifying uncertainty in chemical systems modeling, International journal of chemical kinetics 37 (2005) 368-382.

[10] H. Najm, R. Berry, C. Safta, K. Sargsyan, B. Debusschere, Data-Free Inference of Uncertain Parameters in Chemical Models, Int. J. Uncertain. Quantif. 4 (2014) 111-132.

[11] K. Miki, M. Panesi, E. Prudencio, S. Prudhomme, Probabilistic models and uncertainty quantification for the ionization reaction rate of atomic Nitrogen, J. Comput. Phys. 231 (2012) 3871-3886.

[12] D. Kim, I. El Gharamti, M. Hantouche, A. E. Elwardany, A. Farooq, F. Bisetti, O. Knio, A hierarchical method for Bayesian inference of rate parameters from shock tube data: Application to the study of the reaction of hydroxyl with 2-methylfuran, Combust. Flame 184 (2017) 55-67.

[13] T. Nagy, T. Turányi, Determination of the uncertainty domain of the Arrhenius parameters needed for the investigation of combustion kinetic models, Reliability Engineering \& System Safety 107 (2012) 29-34.

[14] D. A. Sheen, H. Wang, The method of uncertainty quantification and minimization using polynomial chaos expansions, Combust. Flame 158 (2011) 2358-2374.

[15] D. Kim, F. Rizzi, K. W. Cheng, J. Han, F. Bisetti, O. M. Knio, Uncertainty quantification of ion chemistry in lean and stoichiometric homogenous mixtures of methane, oxygen, and argon, Combust. Flame 162 (2015) $2904-2915$.

[16] F. Bisetti, D. Kim, O. Knio, Q. Long, R. Tempone, Optimal Bayesian Experimental Design for Priors of Compact Support with Application to Shock-Tube Experiments for Combustion Kinetics, Int. J. Numer. Meth. Eng. 108 (2016) $136-155$.

[17] R. Malpica Galassi, M. Valorani, H. Najm, C. Safta, M. Khalil, P. Ciottoli, Chemical model reduction under uncertainty, Combustion and Flame 179 (2017) 242-252.

[18] T. Turányi, Sensitivity analysis of complex kinetic systems. Tools and applications, Journal of Mathematical Chemistry 5 (1990) 203-248.

[19] T. Turányi, Applications of sensitivity analysis to combustion chemistry, Reliab. Eng. Syst. Safe. 57 (1997) $41-48$.

[20] T. Turányi, L. Zalotai, S. Dóbé, T. Bérces, Effect of the uncertainty of kinetic and thermodynamic data on methane flame simulation results, Physical Chemistry Chemical Physics 4 (2002) 2568-2578.

[21] I. G. Zsély, J. Zádor, T. Turányi, Uncertainty analysis of updated hydrogen and carbon monoxide oxidation mechanisms, Proceedings of the Combustion Institute 30 (2005) 1273-1281.

[22] A. S. Tomlin, The role of sensitivity and uncertainty analysis in combustion modelling, Proc. Combust. Inst. 34 (2013) 159-176.

[23] P. Constantine, Active Subspaces, Society for Industrial and Applied Mathematics, Philadelphia, PA, 2015.

[24] P. Constantine, P. Diaz, Global sensitivity metrics from active subspaces, Reliab. Eng. Syst. Saf. 162 (2017) 1-13.

[25] M. Vohra, A. Alexanderian, C. Safta, S. Mahadevan, Sensitivity-Driven Adaptive Construction of Reduced-space Surrogates, Journal of Scientific Computing 79 (2019) 1335-1359. 
[26] M. Vohra, A. Alexanderian, H. Guy, S. Mahadevan, Active subspace-based dimension reduction for chemical kinetics applications with epistemic uncertainty, Combustion and Flame (2019) 152-161.

[27] J. Prager, H. N. Najm, J. Zador, Uncertainty quantification in the ab initio rate-coefficient calculation for the $\mathrm{CH}_{3} \mathrm{CH}(\mathrm{OH}) \mathrm{CH}_{3}+\mathrm{OH} \longrightarrow \mathrm{CH}_{3} \mathrm{C}(\mathrm{OH}) \mathrm{CH}_{3}+\mathrm{H}_{2} \mathrm{O}$ reaction, Proc. Combust. Inst. 34 (2013) 583-590.

[28] S. Lee, S. Song, A rapid compression machine study of hydrogen effects on the ignition delay times of n-butane at low-to-intermediate temperatures, Fuel 266 (2020) 116895.

[29] S. Karimkashi, H. Kahila, O. Kaario, M. Larmi, V. Vuorinen, Numerical study on tri-fuel combustion: Ignition properties of hydrogen-enriched methane-diesel and methanol-diesel mixtures, International Journal of Hydrogen Energy (2020).

[30] Y. Wu, M. Zhu, T. Ye, T. Zhou, P. Tang, Numerical simulation of the effects of evaporation on the n-heptane/air autoignition process under different initial air temperatures, Fuel 243 (2019) 202-209.

[31] Y. Zhang, J. Fu, J. Shu, M. Xie, J. Liu, T. Jiang, Z. Peng, B. Deng, Numerical study on auto-ignition characteristics of hydrogen-enriched methane under engine-relevant conditions, Energy Conversion and Management 200 (2019) 112092.

[32] S. Lapointe, C. L. Druzgalski, M. J. McNenly, Numerical study of a micro flow reactor at engine pressures: Flames with repetitive extinction and ignition and simulations with a reduced chemical model, Combustion and Flame 197 (2018) $102-110$.

[33] Z. Yang, X. Li, Z. Feng, L. Lu, Sensitivity analysis and chemical reaction mechanism simplification of blast furnace gas in gas turbine combustor environment, Journal of Mechanical Science and Technology 31 (2017) 2005-2014.

[34] R. Kee, F. M. Rupley, J. A. Miller, M. Coltrin, J. Grcar, E. Meeks, CHEMKIN-PRO, 15105, Reaction Design, San Diego, 2010.

[35] D. G. Goodwin, Cantera C++ users guide, California Institute of Technology (2002).

[36] C. Safta, H. Najm, O. Knio, TChem - A Software Toolkit for the Analysis of Complex Kinetic Models, Technical Report Report No. SAND2011-3282, Sandia National Laboratories, Livermore, CA, USA, 2011.

[37] A. E. Lutz, R. J. Kee, J. A. Miller, SENKIN: A FORTRAN program for predicting homogeneous gas phase chemical kinetics with sensitivity analysis, Technical Report, Sandia National Labs., Livermore, CA (USA), 1988.

[38] W. Ji, Z. Ren, C. Law, Evolution of sensitivity directions during autoignition, Proceedings of the Combustion Institute 37 (2019) $807-815$.

[39] M. Lemke, L. Cai, J. Reiss, H. Pitsch, J. Sesterhenn, Adjoint-based sensitivity analysis of quantities of interest of complex combustion models, Combustion Theory and Modelling 23 (2019) 180-196.

[40] V. Gururajan, F. Egolfopoulos, Direct sensitivity analysis for ignition delay times, Combustion and Flame 209 (2019) 478 -480 .

[41] R. J. Kee, F. M. Rupley, J. A. Miller, The Chemkin Thermodynamic Data Base, Technical Report Report No. SAND-878215B, Sandia National Laboratories, Livermore, CA, USA, 1990.

[42] B. J. McBride, S. Gordon, M. A. Reno, Coefficients for Calculating Thermodynamic and Transport Properties of Individual Species, Technical Report NASA TM-4513, NASA, 1993.

[43] D. Kim, I. El Gharamti, M. Hantouche, A. E. Elwardany, A. Farooq, F. Bisetti, O. Knio, A hierarchical method for Bayesian inference of rate parameters from shock tube data: Application to the study of the reaction of hydroxyl with 2-methylfuran, Combustion and Flame 184 (2017) 55-67.

[44] M. Hantouche, S. M. Sarathy, O. M. Knio, Global sensitivity analysis of n-butanol reaction kinetics using rate rules, Combustion and Flame 196 (2018) 452-465.

[45] P. Brown, G. Byrne, A. Hindmarsh, VODE: A Variable Coefficient ODE Solver, SIAM J. Sci. Stat. Comput. 10 (1989) $1038-1051$

[46] S. Cohen, A. Hindmarsh, CVODE, a Stiff/Nonstiff ODE Solver in C, Computers in Physics 10 (1996) $138-143$.

[47] G. Guennebaud, B. Jacob, et al., Eigen v3, http://eigen.tuxfamily.org, 2010.

[48] R. Yetter, F. Dryer, H. Rabitz, A comprehensive reaction mechanism for carbon monoxide/hydrogen/oxygen kinetics, Combustion Science and Technology 79 (1991) 97-128.

[49] M. Mehl, H. Curran, W. Pitz, C. Westbrook, iso-octane, version 3, https://combustion.llnl.gov/mechanisms/alkanes/isooctane-version-3, 2009.

[50] M. Mehl, H. J. Curran, W. J. Pitz, C. K. Westbrook, Chemical kinetic modeling of component mixtures relevant to gasoline, https://www.osti.gov/biblio/952084, 2009.

[51] M. Mehl, W. Pitz, M. Sjöberg, J. E. Dec, in: SAE Technical Paper 2009-01-1806.

[52] S. M. Sarathy, P. Oßwald, N. Hansen, K. Kohse-Höinghaus, Alcohol combustion chemistry, Prog. Energy Combust. Sci. 44 (2014) 40-102.

[53] J. Prager, H. Najm, K. Sargsyan, C. Safta, W. Pitz, Uncertainty quantification of reaction mechanisms accounting for correlations introduced by rate rules and fitted Arrhenius parameters, Combust. Flame 160 (2013) $1583-1593$. 